\title{
Orthogonality Properties of Iterated Polynomial Mappings
}

\author{
D. Bessis and P. Moussa \\ Service de Physique Théorique, CEN-Saclay, F-91191 Gif-sur-Yvette, France
}

\begin{abstract}
We consider a measure defined on a complex contour and its associated orthogonal polynomials. The action of a polynomial transformation on the measure and the transformation laws of the corresponding orthogonal polynomials are given. Iterating the transformation provides an invariant measure, whose support is the Julia set corresponding to the polynomial transformation. It appears that, up to a constant, the iterated polynomials generated by the initial mapping form a subset of the invariant set of orthogonal polynomials, which fulfill a three term recursion relation. An algorithm is given to compute the coefficients of this recursion relation, which can be interpreted as a linear extension of the iterative procedure.
\end{abstract}

\section{Introduction}

The connection between dynamical systems and iterative maps has received a great deal of attention in the past ten years, initiating a new approach to turbulence [1]. The present situation is best summarized in a review article by Eckmann [2]. In particular one dimensional mappings display intriguing properties under iteration [3], as was already noticed by May [4]. Universality properties of one dimensional mappings have been emphasized by Feigenbaum [5], and the corresponding effects observed in experiments [6]. Although more general mappings display also some universality properties [7], the most useful transformations are those who behave in a regular way in the vicinity of their critical point. Special examples of this situation are provided by polynomial mappings.

On the other hand, iterations of rational mappings have been considered sixty years ago by Julia [8] and Fatou [9], and studied extensively by Brolin [10]. The quadratic polynomials have been considered by Myrberg [11], and recently by Douady and Hubbard [12]. A complete understanding of the iterations of a polynomial mapping seems to require the extension from real to complex analysis. There is in fact a relation between mappings and invariant measures [13], and it is therefore interesting to study polynomial transformations on measures. 
In a paper devoted to a completely different problem, namely the classification of measures having integer moments [14], were considered some properties of polynomial mappings [15], which lead to a connection between iterated mappings and orthogonal polynomials. More precisely, a close algebraic relation between orthogonal polynomials with respect to a measure, and iterated quadratic mappings has been established $[16,17]$. The measure is simply associated to a contour integral on the Julia set [18] linked to the quadratic transformation. Besides the unexpected algebraic relation between iterated maps and orthogonal polynomials, the recursion relation between three consecutive polynomials belonging to the same orthogonal set provides a linear extension of the nonlinear iterative procedure [17]. This linear extension is interpreted as a difference equation of order 2 , that is a discrete version of a second order differential equation with variable coefficients [23]. Of course the difficulty is now to study the properties of these coefficients, an analysis which has been completed in the quadratic case [17].

The aim of this paper is to give a generalization of these results to arbitrary polynomials. The paper is organised as follows: In Sect. II, we recall some properties of orthogonal polynomials, in a somewhat unusual way, the integration being now along a contour in the complex plane of the variable $v$, with a "measure" $\gamma(v)$ analytic on the contour. In Sect. III, we perform polynomial transformations on the measure. In Sect. IV, we establish the relation between two sets of orthogonal polynomials, the second being orthogonal with respect to a measure obtained after a polynomial transformation on the measure of the first set. In Sect. V, we introduce the iteration of the transformation, and give the laws of transformation of orthogonal polynomials under these iterated mappings. In Sect. VI, it is shown that an infinite iteration procedure leads to an invariant measure with respect to the polynomial transformation. By contour integration deformation one reaches the Julia set corresponding to the considered polynomial mapping. Section VII is devoted to the invariant orthogonal polynomials, and it is shown that the iterated polynomials generated by the initial mapping are, up to a constant, a subset of the invariant orthogonal polynomials. In Sect. VIII we briefly recall our preceding results for the quadratic polynomials case, showing that, in this special case, a simple relation exists between isotropic orthogonal systems, that is systems which admit zero norm for some polynomials, and mappings with superstable fixed points. In the conclusion, Sect. IX, we discuss how the recursion relation between invariant orthogonal polynomials appears as a linear extension of the nonlinear iteration procedure. Finally, technical details relevant to Sect. IV are given in an appendix.

Throughout this paper, we shall call "monic" a polynomial of degree $d$ having its highest order term equal to $z^{d}$, and we let $S_{d}$ denote the set of monic polynomials the sum of whose roots is zero, i.e., those with no $z^{d-1}$ term.

\section{Orthogonal Polynomials on a Complex Contour}

We consider a function $g(z)$ which is assumed to be analytic in an open domain $\mathscr{D}$ containing $z=0$. We can draw a contour $\Gamma_{0}$ encircling $z=0$ and contained in $\mathscr{D}$. 
For any point $z$ interior to $\Gamma_{0}$, we can write, using the Cauchy theorem:

$$
\begin{gathered}
g(z)=\sum_{n=0}^{\infty} g_{n} z^{n}=\frac{1}{2 i \pi} \oint_{\Gamma_{0}} \frac{g(u) d u}{u-z}, \\
g_{n}=\frac{1}{2 i \pi} \oint_{I_{0}} \frac{g(u) d u}{u^{n+1}} .
\end{gathered}
$$

The contour $\Gamma_{0}$ has been, as usual, oriented counter-clockwise. Now changing $u$ into $v=\frac{1}{u}$, we get:

$$
\begin{aligned}
& g(z)=\frac{1}{2 i \pi} \oint_{\Gamma_{\infty}} \frac{\gamma(v) d v}{1-v z}, \\
& g(n)=\frac{1}{2 i \pi} \oint_{\Gamma_{\infty}} \gamma(v) v^{n} d v,
\end{aligned}
$$

where $\gamma(v)$ is defined by:

$$
\gamma(v)=\frac{1}{v} g\left(\frac{1}{v}\right) .
$$

Now $\Gamma_{\infty}$ is a contour near infinity, also counter-clockwise oriented, contained in the image of $\mathscr{D}$ through the transformation $v=\frac{1}{u}$, and $z$ is supposed to lie outside the contour $\Gamma_{\infty}$. Therefore $g(z)$ can be considered as the generating function of the $g_{n}$, which appear through (II.4) to be nothing but the moments of the measure $\gamma(v)$ on the contour $\Gamma_{\infty}$. The analyticity properties of $g(z)$ allow us to move the contour as long as no singularity is reached. This extension of the moment problem is a straightforward generalization of the standard moment problem of a measure on the real line: when $g(z)$ is real analytic and has all its singularities localized on the real axis, a small interval containing $z=0$ excluded, the contour $\Gamma_{\infty}$ can be moved in such a way that it encircles the real axis where all singularities of $\gamma(v)$ lie. We get:

where now:

$$
\begin{gathered}
g(z)=\int \frac{\sigma(v) d v}{1-v z}, \\
g_{n}=\int v^{n} \sigma(v) d v,
\end{gathered}
$$

$$
\sigma(v)=\frac{1}{2 i \pi} \lim _{\varepsilon \rightarrow 0}\{\gamma(v-i \varepsilon)-\gamma(v+i \varepsilon)\}
$$

Going back to the general case, we introduce the set of polynomials $P_{n}(z)$ orthogonal with respect to the following scalar product, defined as a contour integration on $\Gamma_{\infty}$ :

$$
\left\langle P_{n}, P_{m}\right\rangle=\frac{1}{2 i \pi} \oint_{\Gamma_{\infty}} P_{n}(v) P_{m}(v) \gamma(v) d v=h_{n} \delta_{m n} .
$$

Here $P_{n}(v)$ is a monic polynomial of degree $n$, which means that the highest degree coefficient is equal to 1 :

$$
P_{n}(v)=v^{n}+a_{1}^{(n)} v^{n-1}+\ldots
$$


Equations (II.9) and (II.10) define in general a unique set of polynomials [19], which have the following explicit representation in terms of the moments $g_{n}$ :

$$
P_{n}(v)=\frac{1}{D_{n}}\left|\begin{array}{llll}
g_{0} & g_{1} & \ldots & g_{n} \\
g_{1} & g_{2} & \ldots & g_{n+1} \\
\vdots & \vdots & & \vdots \\
g_{n-1} & g_{n} & \ldots & g_{2 n-1} \\
1 & v & \ldots & v^{n}
\end{array}\right|
$$

We use $D_{n}$ defined for $n \geqq 1$ as :

$$
D_{n}=\left|\begin{array}{llll}
g_{0} & g_{1} & \ldots & g_{n-1} \\
g_{1} & g_{2} & \ldots & g_{n} \\
\vdots & \vdots & & \vdots \\
g_{n-1} & g_{n} & \ldots & g_{2 n-2}
\end{array}\right| .
$$

Using these definitions, we have the following expression [19] for the "norm" parameter $h_{n}$ :

$$
h_{n}=\frac{D_{n+1}}{D_{n}}, \quad h_{0}=g_{0} .
$$

Existence and uniqueness for the set of polynomials are guaranteed as long as all determinants $D_{n}$ are different from zero, which is the generic situation. The polynomial $P_{n}$ can equivalently be defined as the unique monic polynomial of degree $n$, which is orthogonal to all monomials $v^{p}$, for $p$ less than $n$. In fact we have for any $p \leqq n$ :

$$
\left\langle P_{n}, v^{p}\right\rangle=\frac{1}{2 i \pi} \oint_{\Gamma_{\infty}} P_{n}(v) v^{p} \gamma(v) d v=h_{n} \delta_{n p} .
$$

The set of polynomials $P_{n}$ satisfy the well known three term recursion relation [19], for $n \geqq 2$ :

$$
P_{n}(v)=\left(v-B_{n}\right) P_{n-1}(v)-R_{n} P_{n-2}(v) .
$$

In order to establish this relation, we expand $v P_{n-1}$ as a linear combination of the polynomials $P_{m}(v)$ :

$$
v P_{n-1}(v)=\sum_{k=0}^{n} \alpha_{k}^{(n)} P_{k}(v) .
$$

The orthogonality properties show that:

$$
\left\langle v P_{n-1}, P_{m}\right\rangle=h_{m} \alpha_{m}^{(n)} .
$$

However :

$$
\left\langle v P_{n-1}, P_{m}\right\rangle=\left\langle P_{n-1}, v P_{m}\right\rangle,
$$

which shows that $\alpha_{m}^{(n)}$ is equal to zero if $m<n-2$. On the other hand, comparison of highest order terms in (II.16) shows that:

$$
\alpha_{n}^{(n)}=1, \quad h_{n}=\left\langle v P_{n-1}, P_{n}\right\rangle,
$$


and

$$
\begin{aligned}
& h_{n-1} \alpha_{n-1}^{(n)}=\left\langle v P_{n-1}, P_{n-1}\right\rangle, \\
& h_{n-2} \alpha_{n-2}^{(n)}=\left\langle v P_{n-1}, P_{n-2}\right\rangle=\left\langle v P_{n-2}, P_{n-1}\right\rangle .
\end{aligned}
$$

In view of (II.19), we get:

$$
h_{n-2} \alpha_{n-2}^{(n)}=h_{n-1} .
$$

Introducing the notations

$$
B_{n}=\alpha_{n-1}^{(n)}, \quad R_{n}=\alpha_{n-2}^{(n)},
$$

we get (II.15) with

and

$$
B_{n}=\frac{1}{h_{n-1}}\left\langle v P_{n-1}, P_{n-1}\right\rangle
$$

$$
R_{n}=\frac{h_{n-1}}{h_{n-2}}=\frac{D_{n} D_{n-2}}{\left(D_{n-1}\right)^{2}} .
$$

Of course these equations make sense only in the generic case where all $h_{n}$ are different from zero, and they generalize to a contour $\Gamma_{\infty}$ the classical three term recursion relation for orthogonal polynomials on the real line. Finally note that the generalized scalar product considered here is not the usual hermitian one and it is not assumed to define a positive norm.

\section{Polynomial Transformation on a Measure}

Given an arbitrary polynomial $F(z)$ of degree $d$, we propose to investigate the effect of a change of variable defined by $F$ on the measure $\gamma(v)$. We first consider the transformation: $g(z) \rightarrow g^{F}(z)$ defined by:

$$
g^{F}(z)=\frac{1}{z d} \frac{F^{\prime}(1 / z)}{F(1 / z)} g\left(\frac{1}{F(1 / z)}\right) .
$$

In this equation, $F^{\prime}(1 / z)$ must be understood as $\frac{d}{d u} F(u)$ taken for the value $u=1 / z$. The reason for introducing expression (III.1) becomes clear when reexpressed on the measure density $\gamma(v)$ :

$$
\gamma(v)=\frac{1}{v} g\left(\frac{1}{v}\right), \quad \gamma^{F}(v)=\frac{1}{v} g^{F}\left(\frac{1}{v}\right) .
$$

We get from (III.1):

$$
\gamma^{F}(v)=\frac{1}{d} F^{\prime}(v) \gamma(F(v))
$$

Setting now

$$
\gamma(v)=-\frac{d G(v)}{d v}, \quad \gamma^{F}(v)=-\frac{d G^{F}(v)}{d v},
$$


we get

$$
G^{F}(v)=\frac{1}{d} G(F(v))+\text { const. }
$$

This last equation requires a comment: we have already assumed that $g(z)$ is analytic in the vicinity of zero, then Eq. (III.1) shows that $g^{F}(z)$ is also analytic in the vicinity of zero, because the mapping $z \rightarrow \frac{1}{F(1 / z)}$ preserves zero. If $g(z)$ is
expanded around zero:

$$
g(z)=\sum_{n=0}^{\infty} g_{n} z^{n}
$$

We get a corresponding expansion of $\gamma$ near infinity:

$$
\gamma(v)=\frac{g_{0}}{v}+\frac{g_{1}}{v^{2}}+\ldots+\frac{g_{n}}{v^{n+1}}+\ldots
$$

Integrating (III.7) shows that $G(v)$ has a logarithmic singularity at infinity:

$$
G(v)=-g_{0} \ln v+C+\frac{g_{1}}{v}+\ldots+\frac{g_{n}}{n v^{n}} .
$$

As a consequence, the additional constant in (III.5) depends on which sheets both sides of the equation are considered. It is possible to clarify the situation when $v$ is large enough in modulus. Assuming $F(v)$ to be monic, the constant in (III.5) can be chosen as if $G(v) \sim-g_{0} \ln v, F(v) \sim v^{d}$ when $v \rightarrow \infty$. More precisely, we define the logarithmic function as:

$$
\ln v=\ln \varrho+i \theta \quad \text { when } \quad v=\varrho e^{i \theta} \quad \text { with } \quad-\pi<\theta \leqq \pi .
$$

With the previous convention, we get

$$
\ln v=\frac{1}{d} \ln \left(v^{d}\right)+\frac{2 i k \pi}{d},
$$

where $k$ is an integer satisfying

$$
-\pi<d \theta-2 k \pi \leqq \pi
$$

With this definition of $k$, we can fix the determination of $G(v)$ such that for $|v|$ large enough, we have

$$
G^{F}(v)=\frac{1}{d} G(F(v))-\frac{2 i k \pi}{d} g_{0}
$$

while (III.1) insures that $g(0)=g^{F}(0)=g_{0}$. The validity of (III.12) can be extended to any domain containing the point at infinity, but containing no roots of $F$ and no singularity of $\gamma(v)$. We shall need a modified form of (III.12). Let us define the $d$ inverse functions:

$$
F_{j}^{-1}(v), \quad j=1,2, \ldots, d,
$$

as the $d$ roots of the algebraic equation:

$$
F\left(F_{j}^{-1}(v)\right)=v
$$


Replacing $v$ by $F_{j}^{-1}(v)$ tells us that there exist integers $\tilde{k}_{j}$ such that:

which gives

$$
G^{F}\left(F_{j}^{-1}(v)\right)=\frac{1}{d} G(v)-\frac{2 i \pi \tilde{k}_{j}}{d} g_{0}
$$

$$
\sum_{j=1}^{d} G^{F}\left(F_{j}^{-1}(v)\right)=G(v)-\frac{2 i \pi}{d}\left(\sum_{j} \tilde{k}_{j}\right) g_{0} .
$$

Asymptotically in $v, G$ reduces to $-\ln v$, and $F_{j}^{-1}(v)$ to the different determinations of $v^{1 / d}$. A short calculation shows that $\left(\sum_{j} \tilde{k}_{j}\right)$ vanishes when $d$ is odd, and is equal to \pm 1 according to the sign of $\theta$ when $d$ is even [see (III.9)]. We summarize all this by writing in the neighborhood of infinity:

Theorem 1. For $|v|$ large enough:

where

$$
\sum_{j=1}^{d} G^{F}\left(F_{j}^{-1}(v)\right)=G(v)-\frac{2 i \pi}{d} \eta g_{0}
$$

$$
\begin{array}{ll}
\eta=0 & \text { if } d \text { is odd } \\
\eta=\operatorname{sign}\{\operatorname{Im} v\} & \text { if } d \text { is even. }
\end{array}
$$

Equation (III.16) displays the transformation laws of the integrated measure. In addition we have:

$$
G^{F}\left(F_{j}^{-1}(v)\right)-G^{F}\left(F_{l}^{-1}(v)\right)=\frac{2 i \pi}{d}\left(\tilde{k}_{l}-\tilde{k}_{j}\right) g_{0} .
$$

This last equation expresses the symmetry properties of $G^{F}$ under the exchange of determinations of the inverse of $F$. The ambiguity removing terms appear to be particularly useful when it is possible to squeeze the contour of integration on the real line, as discussed in Eqs. (II.6) and (II.8).

Going back to the measure density transformation, we rewrite (III.3):

$$
\gamma^{F}(v)=\frac{1}{d} F^{\prime}(v) \gamma(F(v))
$$

Replacing $v$ by $F_{j}^{-1}(v)$ yields

$$
\gamma^{F}\left(F_{j}^{-1}(v)\right)=\frac{1}{d} F^{\prime}\left(F_{j}^{-1}(v)\right) \gamma(v)
$$

which can be written as

$$
\gamma^{F}\left(F_{j}^{-1}(v)\right)=\frac{\gamma(v)}{d} \frac{1}{\frac{d}{d v}\left(F_{j}^{-1}(v)\right)}
$$

This last relation enables us to perform a change of variables in a typical contour integral: for arbitrary functions $\phi(v)$ and $\psi(v)$ analytic around infinity, we have:

$$
\frac{1}{2 i \pi} \oint_{\Gamma_{\infty}} \Phi(F(v)) \psi(v) \gamma^{F}(v) d v=\frac{1}{2 i \pi} \oint_{\Gamma_{\infty}} \Phi(v) \frac{1}{d}\left(\sum_{j} \psi\left(F_{j}^{-1}(v)\right)\right) \gamma(v) d v .
$$


This equation is obtained by first changing $v$ into $F_{j}^{-1}(v)$, for a given $j$, in the left hand side, and then observing that the contour has to perform $d$ turns around infinity, but to each turn corresponds a different determination of the inverse. Therefore one can express the result as given in (III.21).

\section{Transformation Laws for the Corresponding Orthogonal Polynomials}

We want to establish a connection between the polynomials $P_{n}(v)$ orthogonal with respect to $\gamma(v)$, and the polynomials $P_{n}^{F}(v)$ orthogonal with respect to $\gamma^{F}(v)$. Consider the integral:

$$
I_{p}=\frac{1}{2 i \pi} \oint_{\Gamma_{\infty}} P_{n}(F(v)) v^{p} \gamma^{F}(v) d v,
$$

where $p$ is any integer smaller or equal to $d n$. According to (III.21), this integral can be equivalently written as:

We set

$$
I_{p}=\frac{1}{2 i \pi} \oint_{\Gamma_{\infty}} P_{n}(v) \frac{1}{d}\left(\sum_{j}\left(F_{j}^{-1}(v)\right)^{p}\right) \gamma(v) d v .
$$

$$
S_{p}(v)=\frac{1}{d} \sum_{j}\left(F_{j}^{-1}(v)\right)^{p} .
$$

We need the following lemma:

Lemma 1. The arithmetic mean $S_{n}(v)$ of the $p^{\text {th }}$ power of the roots of the algebraic equation in $u: F(u)=v$, is a polynomial in $v$ of degree $k$, where $k$ is the largest integer smaller than or equal to $p / d$. When $p / d$ is integer, $S_{p}(v)$ is monic, assuming $F(v)$ to be monic.

In order to prove the lemma, we start from the identity

$$
\frac{F^{\prime}(u)}{F(u)-v}=\sum_{j=1}^{d} \frac{1}{u-F_{j}^{-1}(v)},
$$

changing $u$ into $1 / u$, we get

$$
\frac{1}{d} \frac{F^{\prime}(1 / u)}{u F(1 / u)-u v}=\frac{1}{d} \sum_{j=1}^{d} \frac{1}{1-u F_{j}^{-1}(v)}=\sum_{p=0}^{\infty} u^{p} S_{p}(v),
$$

from which we get:

$$
\sum_{p=0}^{\infty} u^{p} S_{p}(v)=\frac{1}{d} \frac{u^{d-1} F^{\prime}(1 / u)}{u^{d} F(1 / u)} \frac{1}{1-\frac{u^{d} v}{u^{d} F(1 / u)}} .
$$

However, $u^{d-1} F^{\prime}(1 / u)$ and $u^{d} F(1 / u)$ are polynomials and the latter does not vanish at $u=0$ since $F(u)$ is of effective degree $d$. Without computing explicitly, one sees that in the expansion of the right hand side of (IV.6) in powers of $u$, each power of $v$ will arise together with $u^{d}$. This tells us that in $S_{p}(v)$, powers of $v$ of order $r$ such that $d r \leqq p$ can only occur. Therefore the degree of $S_{p}(v)$ is the largest integer smaller than or equal to $p / d$. If $p=d r$, with $r$ integer, there is only one term in $u^{p} v^{r}=\left(u^{d} v\right)^{r}$ in 
the expansion, obtained considering the values of $u^{d} F(1 / u)$ and $u^{d-1} F^{\prime}(1 / u)$ at $u=0$. If $F$ is monic, $u^{d} F(1 / u)$ tends to 1 and $u^{d-1} F^{\prime}(1 / u)$ to $d$ when $u$ tends to zero, which proves that $S_{d r}(v)$ is itself monic, hence completes the proof of the lemma.

In the sequel we shall assume that $F(v)$ is monic. We now come back to (IV.2), and using the previous lemma taking into account (II.14), we get:

$$
\begin{array}{lll}
I_{p}=0 & \text { if } & p<d n \\
I_{p}=h_{n} & \text { if } & p=d n .
\end{array}
$$

Therefore we have the following:

Theorem 2. Let $F(v)$ be a monic polynomial of degree $v$, and let $P_{n}(v)$ (respectively, $P_{n}^{F}(v)$ ) denote the sequence of polynomials orthogonal with respect to $\gamma(v)$ (respectively, $\gamma^{F}(v)$ ). Then:

$$
\begin{gathered}
P_{n}(F(v))=P_{n d}^{F}(v), \\
h_{n}=h_{n d}^{F} .
\end{gathered}
$$

The situation can be summarized as follows : starting from $\gamma(v)$, we generate the set of polynomials $P_{n}(v)$. Through (IV.8) we obtain a subset of polynomials $P_{n}^{F}(v)$ orthogonal with respect to $\gamma^{F}(v)$, namely those for which $n$ is a multiple of $d$. We want to sketch a procedure for computing the missing polynomials and in order to achieve our goal, we will show how to compute the recursion relation coefficients relevant to the polynomials $P_{n}^{F}(v)$. This recursion relation can be used to compute the missing polynomials.

In fact, we first establish a linear three term relation between $P_{n d+d}, P_{n d}, P_{n d-d}$, which are three orthogonal polynomials belonging to any arbitrary complete set of orthogonal polynomials. Starting from the usual relation (II.15):

$$
P_{k}(v)=\left(v-B_{k}\right) P_{k-1}(v)-R_{k} P_{k-2}(v), \quad k \geqq 2,
$$

we introduce a few notations:

$$
A_{k}=v-B_{k}, \quad k \geqq 2 .
$$

We define $M(p, q)$ as the truncated Jacobi tridiagonal matrix, with the following properties :

i) $M(p, q)$ is a square matrix of dimension $q$,

ii) The diagonal elements are:

$$
[M(p, q)]_{i i}=A_{(p+i)}, \quad i=1,2, \ldots, q,
$$

iii) Elements of $M$ belonging to the nearest lines parallel to the diagonal are given by:

$$
\begin{array}{ll}
{[M(p, q)]_{i, i+1}=-1,} & i=1,2, \ldots,(q-1) \\
{[M(p, q)]_{i+1, i}=-R_{(p+i+1)},} & i=1,2, \ldots,(q-1),
\end{array}
$$

iv) All other matrix elements of $M(p, q)$ are equal to zero. We also define the determinant

$$
\Delta(p, q)=\operatorname{det}[M(p, q)]
$$


Here $\Delta(p, q)$ is a polynomial of degree $q$ in the variable $v$. Exceptional cases are

$$
\begin{aligned}
& \Delta(p, 1)=A_{p+1}, \\
& \Delta(p, 0)=1 .
\end{aligned}
$$

With the previous notations, we have the following:

Theorem 3. Given $d \geqq 2$ and $n \geqq 1$, a three term linear relation holds between the polynomials $P_{d n+d}, P_{d n}, P_{d n-d}$, belonging to a complete set of orthogonal polynomials:

$$
\alpha_{n}(v) P_{d n+d}(v)=\beta_{n}(v) P_{d n}(v)+\gamma_{n}(v) P_{d n-d}(v),
$$

where $\alpha_{n}, \beta_{n}, \gamma_{n}$ are monic polynomials in $v$ of respective degrees $d-1,2 d-1, d-1$, the explicit expression of which are given by:

$$
\begin{aligned}
\alpha_{n}(v)= & \Delta(d n-d+1, d-1), \\
\beta_{n}(v)= & \Delta(d n-d+1, d-1) \Delta(d n, d)-R_{d n+1} \Delta(d n-d+1, d-2) \\
& \cdot \Delta(d n+1, d-1), \\
\gamma_{n}(v)= & R_{d n-d+2} R_{d n-d+1} \ldots R_{d n+1} \Delta(d n+1, d-1) .
\end{aligned}
$$

Equations (IV.17) and (IV.20) can even be extended to the case $d=1$ provided we perform the identification $\Delta(p,-1)=0$. The proof of Theorem 3 is given in the appendix, and it will make use of the following identity, which results from the definition (IV.14):

$$
\Delta(p, q)=A_{p+1} \Delta(p+1, q-1)-R_{p+2} \Delta(p+2, q-2) .
$$

Consider now the two sequences of polynomials $P_{n}(v)$ and $P_{n}^{F}(v)$, introduced at the beginning of this section. We have

$$
\begin{aligned}
& P_{n}(v)=A_{n} P_{n-1}(v)-R_{n} P_{n-2}(v), \\
& P_{n}^{F}(v)=A_{n}^{F} P_{n-1}^{F}(v)-R_{n}^{F} P_{n-2}(v) .
\end{aligned}
$$

Now changing $v$ into $F(v)$ in (IV.22), and using (IV.8), we get

$$
P_{n d+d}^{F}(v)=\left(F(v)-B_{n+1}\right) P_{n d}^{F}(v)-R_{n+1} P_{n d-d}^{F}(v) .
$$

An expression for the set of polynomials $P_{n}^{F}$ similar to (IV.17) can be written by replacing all $\Delta$ by $\Delta^{F}$, where the superscript $F$ means that in (IV.11), (IV.13), and (IV.15), $A_{n}, B_{n}$, asd $R_{n}$ have to be replaced by the corresponding $A_{n}^{F}, B_{n}^{F}$, and $R_{n}^{F}$. The expression of $P_{n d+d}^{F}$ thus obtained can be equated to (IV.24) and we get the relation

$$
Q_{(2 d-1)} P_{d n}^{F}(v)=S_{(d-1)} P_{d n-d}^{F}(v)
$$

where

$$
\begin{aligned}
Q_{(2 d-1)}(v)= & \Delta^{F}(d n-d+1, d-1)\left[\Delta^{F}(d n, d)-F(v)+B_{n+1}\right] \\
& -\Delta^{F}(d n+1, d-1) \Delta^{F}(d n-d+1, d-2) R_{d n+1}^{F}, \\
S_{(d-1)}(v)= & -R_{n+1} \Delta^{F}(d n-d+1, d-1) \\
& +R_{d n-d+2}^{F} R_{d n-d+1}^{F} \ldots R_{d n+1}^{F} \Delta^{F}(d n+1, d n-1) .
\end{aligned}
$$


The degrees of the polynomials $Q_{(2 d-1)}$ and $S_{(d-1)}$ are respectively $(2 d-1)$ and $(d-1)$. Therefore the degrees of both sides of Eq. (IV.25) do not match, and cancellations have to occur. More precisely the right hand side is of degree at most $(d n-1)$, and in the left hand side $P_{d n}^{F}$ is monic and has degree $d n$. The only possibility is that $S_{(d-1)}$ and $Q_{(2 d-1)}$ vanish identically. Therefore we have

Theorem 4. The coefficients of the three term recursion relation satisfied by the polynomials $P_{n}(v)$ and $P_{n}^{F}(v)$ are connected through the set of polynomial identities in $v$

$$
\begin{gathered}
\Delta^{F}(d(n-1)+1, d-1)=\Delta^{F}(d n+1, d-1), \\
R_{n+1}=R_{n d-d+2}^{F} R_{n d-d+3}^{F} \cdots R_{n d+1}^{F}, \\
F(v)-B_{n+1}=\Delta^{F}(d n, d)-\Delta^{F}(d n-d+1, d-2) R_{d n+1}^{F} .
\end{gathered}
$$

Indeed $S_{(d-1)} \equiv 0$ gives (IV.29) and (IV.28) because the $\Delta$ polynomials are monic. Equation (IV.30) is obtained from $Q_{(2 d-1)} \equiv 0$. Equation (IV.28) is independent of the coefficients $B_{n}$ and $R_{n}$, and expresses a periodicity law of period $d$ for certain combinations of $A_{n}^{F}$ and $R_{n}^{F}$, obtained in expanding (IV.28) in powers of $v$. We set

$$
\Delta^{F}(d n+1, d-1)=v^{d-1}+\sum_{p=0}^{d-2} v^{p} \Gamma_{p}\left(B_{d n+2}^{F}, B_{d n+3}^{F}, \ldots, B_{d n+d}^{F} ; R_{d n+3}^{F}, R_{d n+4}^{F}, \ldots, R_{d n+d}^{F}\right) .
$$

So we have from (IV.28) the theorem:

Theorem 5. The coefficients $B_{m}^{F}$ and $R_{p}^{F}$ satisfy the following periodicity properties: $\Gamma_{p}$ is invariant under the change of $n$ into $(n+1)$.

This periodicity law is related to the exchange of the roots $F_{j}^{-1}(v)$ in the contour integral (IV.1).

In the $d=2$ case, Eqs. (IV.28) and (IV.30) are valid provided we set $\Delta^{F}(p, 0)=1$. In this case we have:

$$
\left.\begin{array}{c}
R_{n+1}=R_{2 n}^{F} R_{2 n+1}^{F}, \\
B_{2 n+2}^{F}=B_{2 n}^{F}, \\
B_{2 n+1}^{F}+B_{2 n+2}^{F}=-\alpha, \\
B_{2 n+1}^{F} B_{2 n+2}^{F}-R_{2 n+1}^{F}-R_{2 n+2}^{F}=\beta-B_{n+1},
\end{array}\right\}
$$

with

$$
F(v)=v^{2}+\alpha v+\beta .
$$

Equations (IV.32) permit the recursive computation of the coefficients $B^{F}$ and $R^{F}$ : knowing $R_{2 n}^{F}$ and $B_{2 n}^{F}$, we get $R_{2 n+1}^{F}, B_{2 n+2}^{F}, B_{2 n+1}^{F}$, and $R_{2 n+2}^{F}$ using the values of $R_{n+1}$ and $B_{n+1}$. We initialize the system by setting:

$$
B_{2}^{F}=-\frac{\alpha}{2}, R_{2}^{F}=-\beta+\frac{\alpha^{2}}{4}+B_{1} .
$$

Initial values $B_{2}^{F}$ and $R_{2}^{F}$ have to be computed directly from (II.11), (II.12), (II.24), and (II.25) using (II.1) and (III.1). 
This recursive algorithm for computing $B_{m}^{F}$ and $R_{p}^{F}$ is not limited to the quadratic case, as is shown by the following theorem, the proof of which is given in the appendix.

Theorem 6. The system of Eqs. (IV.28)-(IV.30) permits the recursive computation of all coefficients $B_{n}^{F}$ and $R_{p}^{F}$ from those contained in $\Delta^{F}(1, d-1)$. The latter ones must be computed directly from (III.1) and (III.2). The recursive procedure is linear, except for Eq. (IV.29) which is used to compute $R_{d n+1}^{F}$ in terms of lower order coefficients. The recursion breaks off if any of the $R_{p}^{F}$ happens to vanish.

We want to emphasize this last result: the computation of the coefficients $R^{F}$ and $B^{F}$ is always made through unambiguous linear or homographic relations which define a unique solution, provided no $R_{p}^{F}$ vanish. In Sect. VII we shall give examples where this is not the case. However in the generic case, the $R^{F}$ will not vanish and we can reconstruct the complete sequence $P_{n}^{F}(v)$ from the initial sequence $P_{n}(v)$ using the recursion relations.

Before ending this section, let us give an operator version of Theorem 2 , as already mentioned in reference [23] for quadratic polynomials. Consider the infinite Jacobi matrix defined by Eqs. (IV.11)-(IV.13), where we let $q$ go to infinity. We define:

$$
H=M(0, \infty) .
$$

Let us denote $\psi(v)$ as the infinite dimensional vector with components

$$
\psi_{n}(v)=P_{n}(v), \quad n=0,1,2, \ldots
$$

By the three term linear recursion relation (IV.9), $\psi(v)$ is an eigenvector of $H$ :

$$
H \psi(v)=v \psi(v) \text {. }
$$

Clearly one can define, in the same way, the infinite Jacobi matrix $H^{F}$ and its corresponding eigenvectors $\psi^{F}(v)$, related to the transformed polynomials $P_{n}^{F}(v)$. We also need the decimation operator $D$ of order $d$, defined as

$$
(D \psi)_{n}=\psi_{(n d)}, \quad n=0,1,2, \ldots
$$

Using this notation, we can rewrite Theorem 2 as

$$
\left(D F\left(H^{F}\right)\right) \psi^{F}(v)=(H D) \psi^{F}(v),
$$

which leads us to the operator valued equation

$$
D F\left(H^{F}\right)=H D \text {. }
$$

It is valid in the topological space spanned by the vectors $\psi^{F}(v)$ when $v$ varies, with a suitable closure condition. It is not necessary to be precise for our purpose here.

\section{Iterations of Polynomial Transformations on a Measure}

In Sect. III we have introduced the transformation:

$$
g^{F}(z)=\frac{1}{z d} \frac{F^{\prime}(1 / z)}{F(1 / z)} g\left(\frac{1}{F(1 / z)}\right) .
$$


To any complex polynomial $F$ of degree $d$ (V.1) associates a transformation $T_{F}$ acting on the set of analytic functions $g(z)$ in the vicinity of zero:

$$
g \stackrel{T_{F}}{\longrightarrow} g^{F} ; \quad\left(T^{F} g\right)(z)=g^{F}(z) .
$$

With the above notations, we have:

Theorem 6. Consider two arbitrary polynomials $F_{1}$ and $F_{2}$ of respective degrees $d_{1}$ and $d_{2}$, and the corresponding transformations $T^{F_{1}}$ and $T^{F_{2}}$. Then:

$$
T^{F_{2}} \circ T^{F_{1}}=T^{\left(F_{1} \circ F_{2}\right)},
$$

where

$$
\left(F_{1} \circ F_{2}\right)(z)=F_{1}\left(F_{2}(z)\right) .
$$

Theorem 6 can be visualized as:

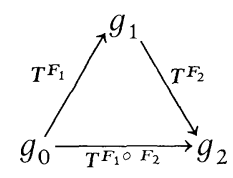

In other words $T^{F}$ is an antirepresentation of the composition law of polynomial mappings. The proof of Theorem 6 can be achieved by straightforward calculation, but Theorem 6 is in fact included in (III.12). As an application, we consider successive iterations of $F$ :

$$
\begin{aligned}
F^{(1)}(z) & =F(z), \\
F^{(n)}(z) & =F\left(F^{(n-1)}(z)\right) .
\end{aligned}
$$

The corresponding iterated transformations, starting from an initial function $g^{(0)}(z)$, are :

$$
\begin{aligned}
& g^{(1)}=T^{F} g^{(0)} \\
& g^{(n)}=T^{F} g^{(n-1)} .
\end{aligned}
$$

From Theorem 6, we deduce the identity:

$$
g^{(n)}=T^{\left(F^{(n)}\right)} g^{(0)},
$$

which can be written as

$$
g^{(n)}(z)=\frac{1}{z d^{n}} \frac{F^{(n)}(1 / z)}{F^{(n)}(1 / z)} g^{(0)}\left(\frac{1}{F^{(n)}(1 / z)}\right),
$$

or equivalently

$$
g^{(n)}(z)=\frac{1}{d^{n}} \frac{z^{\left(d^{n}-1\right)} F^{(n)^{\prime}}(1 / z)}{z^{\left(d^{n}\right)} F^{(n)}(1 / z)} g^{(0)}\left(\frac{z^{\left(d^{n}\right)}}{z^{\left(d^{n}\right)} F^{(n)}(1 / z)}\right) .
$$

From (V.9) we see that the expansion in powers of $z$ of $g^{(n)}$ coincides up to order $z^{\left(d^{n}\right)}$ excluded with the expansion of the rational fraction $R_{(n)}^{F}(z)$ defined by

$$
R_{(n)}^{F}(z)=\frac{1}{d^{n}} \frac{z^{\left(d^{n}-1\right)} F^{(n)^{\prime}}(1 / z)}{z^{\left(d^{n}\right)} F^{(n)}(1 / z)}
$$


multiplied by the constant $g^{(0)}(0)$. Apart from this constant the $d^{n}$ first terms of the expansion of $g^{(n)}(z)$ do not depend on the initial function $g^{(0)}(z)$. More precisely, these terms are determined by the following result:

Theorem 7. The expansion of $g^{(n)}(z)$ coincide up to order $z^{\left(d^{n}\right)}$ excluded, with the series expansion $g^{\infty}(z)$ which is the unique solution, in the sense of formal power series, of the equation

$$
g^{\infty}(z)=\frac{1}{d} \frac{z^{d-1} F^{\prime}(1 / z)}{z^{d} F(1 / z)} g^{\infty}\left(\frac{z^{d}}{z^{d} F(1 / z)}\right),
$$

with the additional condition

$$
g^{\infty}(0)=g^{(0)}(0)
$$

In order to prove the result, we write the formal power series:

$$
g^{\infty}(z)=\sum_{n=0}^{\infty} g_{n}^{\infty} z^{n}
$$

and we expand both sides of (V.11):

$$
\sum_{n=0}^{\infty} g_{n}^{\infty} z^{n}=\frac{1}{d} z^{d-1} F^{\prime}(1 / z) \sum_{n=0}^{\infty} g_{n}^{\infty} \frac{z^{d n}}{\left(z^{d} F(1 / z)\right)^{n+1}} .
$$

Since $\frac{1}{d} z^{d-1} F^{\prime}(1 / z)$ takes the value 1 when $z$ goes to zero, the equation (V.14) does not fix $g_{0}^{\infty}$ which is thus arbitrary. In the right hand side, $g_{n}^{\infty}$ enters only in coefficients of powers $z^{p}$ with $p \geqq d n$. Therefore knowledge of $g_{0}^{\infty}$ allows us to compute $g_{1}^{\infty}, g_{2}^{\infty}, \ldots, g_{d-1}^{\infty}$. Knowing $g_{1}^{\infty}$ permits the computation of $g_{d}, g_{d+1}^{\infty}, \ldots, g_{2 d-1}^{\infty}$, and an obvious recursive argument completes the proof of uniqueness of the solution of (V.11) and (V.12). On the other hand, we could have initiated the recursion (V.7) and (V.9), with $g^{(0)}(z)$ replaced by the formal series $g^{\infty}(z)$ already obtained. Equation (V.9) remains satisfied in the sense of formal power series at least, when in both sides $g^{(0)}(z)$ and $g^{(n)}(z)$ are replaced by $g^{\infty}(z)$. Therefore the expansions of $g^{\infty}(z)$ and $g^{\infty}(0) R_{(n)}^{F}(z)$ coincide up to order $z^{\left(d^{n}\right)}$ excluded. As a consequence, the expansion of $g^{(n)}(z)$ coincide with $g^{(0)}(0) R_{(n)}^{F}(z)$ and $g^{\infty}(z)$ up to the same order, which establishes the theorem. We also have obtained:

Theorem 8. The expansions of $R_{(n)}^{F}(z)$ and $R_{(n+p)}^{F}(z)$ for any $p$ coincide up to order $z^{\left(d^{n}\right)}$ excluded.

We now let $S_{d}$ denote the set of monic complex polynomials of degree $d$ the sum of whose roots vanishes. We shall see that it is possible to restrict ourselves to the class $S_{d}$, and get more precise results than Theorems 7 and 8 .

Consider a linear transformation $L(z)$ and its inverse $L^{-1}(z)$

$$
\left.\begin{array}{rl}
L(z) & =\alpha z+\beta \\
L^{-1}(z) & =\frac{z}{\alpha}-\frac{\beta}{\alpha}
\end{array}\right\}
$$

such that

$$
L\left(L^{-1}(z)\right)=z
$$


To any polynomial $F(z)$ we associate $\Phi(z)$ through a linear Möbius transformation

which makes

$$
\Phi(z)=L\left(F\left(L^{-1}(z)\right)\right)
$$

$$
\begin{aligned}
& \Phi(z)=\alpha F\left(\frac{z}{\alpha}-\frac{\beta}{\alpha}\right)+\beta \\
& F(z)=\frac{1}{\alpha} \Phi(\alpha z+\beta)-\frac{\beta}{\alpha} .
\end{aligned}
$$

These relations can be iterated, and we get

$$
\begin{gathered}
\Phi^{(n)}(z)=L\left(F^{(n)}\left(L^{-1}(z)\right)\right), \\
\Phi^{(n)}(z)=\alpha F^{(n)}\left(\frac{z}{\alpha}-\frac{\beta}{\alpha}\right)+\beta .
\end{gathered}
$$

Given an arbitrary polynomial $\Phi(z)$ it is convenient to choose $\alpha$ and $\beta$ in order to set the highest order term of $F$ equal to 1 , and cancel the term or order $z^{d-1}$. Setting for large $z$ :

and using

$$
\Phi(z)=\phi_{0} z^{d}+\phi_{1} z^{d-1}+0\left(z^{d-2}\right)
$$

$$
\alpha^{d-1}=\frac{1}{\phi_{0}}, \quad \beta=-\frac{1}{d} \frac{\phi_{1}}{\phi_{0}},
$$

we get

$$
F(z)=z^{d}+0\left(z^{d-2}\right) .
$$

Equation (V.22) can always be satisfied when $\alpha$ is allowed to be complex. If we want to restrict ourselves to the real case, assuming $\phi$ real, we can only, keeping $F$ real, ensure that:

$$
F(z)=\operatorname{sign}\left(\phi_{0}\right) z^{d}+0\left(z^{d-2}\right)
$$

when $d$ is odd. There is no restriction when $d$ is even. Therefore we have the following:

Theorem 9. Given any complex polynomial $\Phi(z)$ of degree $d$, there exist $F(z) \in S_{d}$ such that

$$
\phi(z)=\alpha F\left(\frac{z}{\alpha}-\frac{\beta}{\alpha}\right)+\beta
$$

with

$$
\alpha=\phi_{0}^{-\frac{1}{(d-1)}}, \quad \beta=-\frac{1}{d} \frac{\phi_{1}}{\phi_{0}},
$$

and we have

$$
\Phi^{(n)}(z)=\alpha F^{(n)}\left(\frac{z}{\alpha}-\frac{\beta}{\alpha}\right)+\beta
$$

The properties of $\phi^{(n)}(z)$ can then be easily deduced from those of $F^{(n)}(z)$ with $F \in S_{d}$. Notice that all monic odd or even polynomials belong to $S_{d}$. 
We now assume $F \in S_{d}$, and we consider the associated transformation $T^{F}$ introduced (V.1) and (V.2) as well as the rational fraction already introduced in (V.10), which we rewrite as:

$$
R_{(n)}^{F}=T^{F} \cdot 1,
$$

which means that we apply $F$ to the constant function equal to 1 . We also consider $g^{\infty}(z)$ as the unique solution, in the sense of formal power series, of:

$$
\begin{aligned}
& g^{\infty}=T^{F} \cdot g^{\infty}, \\
& g^{\infty}=\sum_{n=0}^{\infty} g_{n}^{\infty} z^{n}, \quad g_{0}^{\infty}=1 .
\end{aligned}
$$

Then we have the following:

Theorem 10. When $F \in S_{d}$, the following properties hold:

i) $g_{1}^{\infty}=0$,

ii) the expansion of $R_{(n)}^{F}$ coincides with $g^{\infty}$ up to order $z^{\left(2 d^{n}\right)}$ excluded, $p \geqq 0$,

iii) the expansion of $R_{(n)}^{F}$ and $R_{(n+p)}^{F}$ coincide up to order $z^{\left(2 d^{n}\right)}$ excluded for any

iv) $R_{(n)}^{F}$ is a $\left[d^{n}-1 / d^{n}\right]$ Padé approximant to the formal power series $g^{\infty}(z)$,

v) $R_{(n)}^{F}$ is a $\left[d^{n}-1 / d^{n}\right]$ Padé approximant to the function $g^{(n)}=T^{\left(F^{(n)}\right)} g^{(0)}$ $=\left(T^{F}\right)^{n} g^{(0)}$, where $g^{(0)}$ is an arbitrary analytic function in the neighborhood of zeroor equivalently a formal power series expansion - such that:

$$
g^{(0)}(z)=\sum_{n=0}^{\infty} g_{n}^{(0)} z^{n}, \quad g_{0}^{(0)}=1, \quad g_{1}^{(0)}=0
$$

Let us prove the theorem: $g_{1}^{\infty}$ is obtained by expanding to first order $g^{\infty}=T^{F} g^{\infty}$, which has the same expansion up to order $z^{\left(d^{n}\right)}$ than $R_{(1)}^{F}$. This gives immediately $g_{1}^{\infty}=0$, since the terms of order $z^{d-1}$ vanish in $F(z)$. Using $g_{1}^{\infty}=0$ shows that $R_{(n)}^{F}$ and $g^{\infty}$ have to coincide now up to order $z^{\left(2 d^{n}\right)}$, as seen from (V.9). The same properties occur immediately for the expansion of $R_{(n)}^{F}$ and $R_{(n+p)}^{F}$. Now (V.28) or (V.10) shows that $R_{(n)}^{F}$ is a rational fraction whose numerator and denominator have respective degree $d^{n}-1$ and $d^{n}$. Since coincidence between expansion of $R_{(n)}^{F}$ and $g^{\infty}$ occur up to $z^{\left(2 d^{n}\right)}$ excluded, $R_{(n)}^{F}$ is a $\left[d^{n}-1 / d^{n}\right]$ Padé approximant [20] to $g^{\infty}$. Item $v$ ) of the theorem goes on through a similar argument, observing that if $g_{0}^{(0)}=1$ and $g_{1}^{(0)}=0$, we have: $g^{(0)}(z)=1+0\left(z^{2}\right)$. Hence the theorem is proven.

Before closing this section, we shall give another algebraic theorem, concerning arbitrary polynomials $F$, not necessarily belonging to $S_{d}$ :

Theorem 11. To any formal power series $g(z)$, let us associate the $[p-1 / p]_{g}$ Pade approximant defined for small $z$ by

$$
g(z)-[p-1 / p]_{g}(z)=0\left(z^{2 p}\right),
$$

where $[p-1 / p]$ denotes a rational fraction in $z$ with numerator and denominator of degree, respectively, $(p-1)$ and $p$. Then for any polynomial $F$ of degree $d$, we have the following identity:

$$
[d p-1 / d p]_{g}(z)=\frac{1}{d z} \frac{F^{\prime}(1 / z)}{F(1 / z)}[p-1 / p]_{g}\left(\frac{1}{F(1 / z)}\right) .
$$



The proof starts from (V.30), in which we change first $z$ into $1 / F(1 / z)=\frac{z^{d}}{z^{d} F(1 / z)}$.
Then we multiply both sides by $R_{(1)}^{F}(z)$ defined in (V.10) and we get:

$$
g^{F}(z)-\psi(z)=0\left(z^{2 d p}\right)
$$

with $\psi(z)$ defined by

$$
\psi(z)=\frac{1}{d z} \frac{F^{\prime}(1 / z)}{F(1 / z)}[p-1 / p]_{g}\left(\frac{1}{F(1 / z)}\right) .
$$

In order to complete the proof we only need to check that the rational fraction $\psi(z)$ has the required degrees in $z: d p-1$ for the numerator, and $d p$ for the denominator. Writing $[p-1 / p]_{g}(z)=\frac{N_{p-1}(z)}{D_{p}(z)}$, we get

$$
\psi_{(z)}=\frac{1}{d z} F^{\prime}(1 / z) \frac{\bar{N}_{p-1}(F(1 / z))}{\bar{D}_{p}(F(1 / z))}=\frac{1}{d} z^{d-1} F^{\prime}(1 / z) \frac{z^{d p-d} \bar{N}_{p-1}(F(1 / z))}{z^{d p} \bar{D}_{p}(F(1 / z))},
$$

where $\bar{D}_{p}(z)=z^{p} D_{p}(1 / z)$ and $\bar{N}_{p-1}(z)=z^{p-1} N_{p-1}(1 / z)$, which shows that $\psi$ has the required degrees.

\section{An Invariant Measure Associated to a Polynomial Mapping}

In the previous section, we have defined a transformation $T^{F}$ acting on the set of analytic functions in the neighbourhood of zero. If we consider now $T^{F}$ as acting on the set of formal power series, we have shown that assuming $g^{(0)}(0)=1,\left(T^{F}\right)^{n} g^{(0)}$ tends to $g^{\infty}(z)$, unique solution of (V.29). Still in the sense of formal power series, the sequence $R_{(n)}^{F}(z)$ tends toward $g^{\infty}(z)$ when $n$ tends to infinity.

We will now make the connection with classical results on iteration of polynomial mappings [10]. For this, we observe that since the expansion of $g^{\infty}(z)$ is obtained as a limit of rational fractions, analyticity of $g^{\infty}(z)$ will follow from Vitali's theorem [21] in any domain D in which we can establish a bound for $R_{(n)}^{F}(z)$ uniformly in $z$ and independent of $n$, for at least an infinite subsequence of integers $n$. But $R_{(n)}^{F}$ has a special form

$$
R_{(n)}^{F}(z)=\frac{1}{d^{n} z} \frac{F^{(n)^{\prime}}(1 / z)}{d^{n} z F^{(n)}(1 / z)}=\frac{1}{d^{n}} \sum_{i=1}^{d^{n}} \frac{1}{1-\sigma_{i}^{(n)} z},
$$

where the $\sigma_{i}$ are the $d^{n}$ roots of the iterated polynomial $F^{(n)}(z)$. Therefore we get

$$
\left|R_{(n)}^{F}(z)\right| \leqq \sup _{i}\left|\frac{1}{1-\sigma_{i}^{(n)} z}\right|
$$

Since $F$ is a polynomial of degree $>1$, there is a constant $\sigma>0$ such that, if $|z|>\sigma$ then $|F(z)| \geqq \sigma$. Hence for any $n$ all the roots of $F^{(n)}$ must have modulus smaller than $\sigma:\left|\sigma_{i}^{(n)}\right|<\sigma$, for all $i$, and $n$. Therefore $\left|R_{(n)}^{F}(z)\right| \leqq 2$ as long as $|z|<\frac{1}{2 \sigma}$, and we can apply Vitali's theorem to the sequence $R_{(n)}^{F}(z), n$ going to infinity, since we have already shown the convergence in the sense of formal power series. We have the following: 
Theorem 12. The function $g^{\infty}(z)$ defined in Theorem 7, Eq. (V.11) is analytic in the vicinity of zero.

$$
\text { To } g^{\infty}(z) \text { corresponds : } \gamma^{\infty}(v)=\frac{1}{v} g^{\infty}\left(\frac{1}{v}\right)
$$

which has the following important properties deduced from (V.11):

$$
\left(\gamma^{\infty}\right)^{F}(v)=\gamma^{\infty}(v)
$$

which shows that $\gamma^{\infty}$ is invariant under the polynomial transformation $F(v)$. This property is best expressed by Eq. (III.21) which now reads :

$$
\frac{1}{2 i \pi} \oint_{\Gamma_{\infty}} \Phi(F(v)) \psi(v) \gamma^{\infty}(v) d v=\frac{1}{2 i \pi} \oint_{\Gamma_{\infty}} \Phi(v) \frac{1}{d}\left(\sum_{j} \psi\left(F_{j}^{-1}(v)\right)\right) \gamma^{\infty}(v) d v,
$$

where $\Phi$ and $\psi$ have the required analyticity properties in the vicinity of the point at infinity. Now the contour of integration can be moved until the singularities of $\gamma^{\infty}(v)$ are reached. But the argument given above in the proof of Theorem 12 makes it almost evident that the singularities of $\gamma^{\infty}(v)$ are accumulation points of the orbit of 0 under iterates of the multivalued inverse function $F^{-1}(z)$. Another classical result, given by Brolin [10] shows that such sequences of iterations will accumulate towards the Julia set associated to the polynomial $F(v)$. All delicate proofs being contained in the classical papers [8-10], we shall not reproduce them here, but notice that for our argument we will only need analyticity of $g^{\infty}(z)$ in the vicinity of zero, and it is clear that the contour integration can be deformed towards the Julia set which in some sense "supports" the measure $\gamma^{\infty}(v)$. The use of the word "measure" may at first slight look improper here and should have been replaced by "boundary value of an analytic function". However in Eq. (VI.1), the rational fraction has only simple poles, even when the roots of the denominator are multiple. Using $1 / z$ as a variable, we see that all the residues in $R_{(n)}^{F}(z)$ are real and positive, their total sum being equal to one. These observations are essential ingredients to maintain control of the limiting process in the approach to the boundary of the analyticity domain and they suggest that the boundary value of $\gamma^{\infty}(v)$ is actually a measure. Of course a complete proof requires additional information on the boundary, i.e. the Julia set. We do not intend to give it here. The connection of our arguments with the classical works mentioned above is made more explicit by considering $\gamma^{\infty}(v)$ as the logarithmic derivative of a function $\psi(v)$

$$
\gamma^{\infty}(v)=\frac{\psi^{\prime}(v)}{\psi(v)} .
$$

There $\psi(v)$ is a solution of the Bottcher equation considered in references $[8,9]$ :

$$
(\psi(v))^{d}=\psi(F(v)) .
$$

\section{The Orthogonal Polynomials Generated by the Invariant Measure}

Consider a polynomial $F \in S_{d}: F$ is monic and the sum of its roots vanishes. Then Theorem 10 applies and we see that $R_{(n)}^{F}$ defined in (V.10) is a $\left[d^{n}-1 / d^{n}\right]$ Padé approximant to $g^{\infty}(z)$ defined in (V.11) with $g^{\infty}(0)=1$. Therefore $z^{\left(d^{n}\right)} F^{(n)}(1 / z)$ is the 
denominator of the $\left[d^{n}-1 / d^{n}\right]$ Padé approximant to $g^{\infty}(z)$, and, according to a classical lemma of Padé approximant theory, belongs to a family of orthogonal polynomials. The lemma is the following:

Lemma 2. Let $\frac{R_{n-1}(z)}{S_{n}(z)}$ be a $[n-1 / n]$ Padé approximant to a function $g(z)$ analytic in the vicinity of zero, then the polynomials $z^{n} S_{n}\left(\frac{1}{z}\right)$ form a set or orthogonal polynomials with respect to $\gamma(z)=\frac{1}{z} g\left(\frac{1}{z}\right)$ and we have

$$
\frac{1}{2 i \pi} \oint_{\Gamma_{\infty}} z^{n} S_{n}\left(\frac{1}{z}\right) z^{m} S_{m}\left(\frac{1}{z}\right) \frac{1}{z} g\left(\frac{1}{z}\right) d z=C_{n} \delta_{m n} .
$$

The proof follows from the definition of Padé approximants [20]:

$$
S_{n}(z) g(z)-R_{n-1}(z)=z^{2 n} \Phi_{n}(z),
$$

where $\Phi_{n}(z)$ is analytic in the neighborhood of zero. Consider the following contour integral around zero:

$$
I=\frac{1}{2 i \pi} \oint_{\Gamma_{0}} S_{n}(z) S_{m}(z) g(z) \frac{d z}{z^{n+m+1}} .
$$

Using (VII.2) we get

$$
I=\frac{1}{2 i \pi} \oint_{\Gamma_{0}} S_{m}(z) R_{n-1}(z) \frac{d z}{z^{n+m+1}}+\frac{1}{2 i \pi} \oint_{\Gamma_{0}} S_{m}(z) \Phi_{n}(z) \frac{d z}{z^{m-n+1}} .
$$

It follows immediately from the residue theorem that the first integral always vanishes, and similarly the second when $n>m$. Symmetry insures that $I$ vanishes also when $m>n$, and we have

$$
I=S_{m}(0) \Phi_{m}(0) \delta_{m n}
$$

Changing $z$ into $\frac{1}{z}$ in the integral $I$ gives (VII.1) which proves the lemma. Using Lemma 2 and remarks given at the opening of this section, we get the following:

Theorem 13. When $F \in S_{d}, F^{(n)}(z)$ is a polynomial of degree $d^{n}$, belonging to the set of orthogonal polynomials with respect to the measure $\gamma^{\infty}(v)=\frac{1}{v} g^{\infty}\left(\frac{1}{v}\right)$.

A straightforward application of Theorem 9 allows us to extend the result in the following:

Theorem 14. Consider any complex polynomial

$$
\Phi(z)=\Phi_{0} z^{d}+\Phi_{1} z^{d-1}+\ldots .
$$

Then the polynomials

$$
\frac{1}{\alpha} \Phi^{(n)}(\beta+\alpha y)-\frac{\beta}{\alpha}, \quad \alpha=\Phi_{0}^{-\frac{1}{(d-1)}}, \quad \beta=-\frac{1}{d} \frac{\phi_{1}}{\phi_{0}}
$$


belong to a family of orthogonal polynomials with respect to a measure $\gamma^{\infty}(v)$ generated by $F \in S_{d}$ and defined by

$$
F(z)=\frac{1}{z} \Phi(\beta+\alpha z)-\frac{\beta}{\alpha} .
$$

This last theorem is connected with Theorem 2, Sect. IV in the following way. From (IV.8) and (VI.4) we see that the orthogonal polynomials with respect to $\gamma^{\infty}(v)$ fulfill the relation

$$
P_{p}(\Phi(v))=P_{d p}(v)
$$

which by iteration gives

$$
P_{p}\left(\Phi^{(n)}(v)\right)=P_{d^{n} p}(v) .
$$

However for $p=1$, we have

$$
P_{1}\left(\Phi^{(n)}(v)\right)=P_{d^{n}}(v) .
$$

It is easy to see that if

$$
\Phi=z^{d}+\Phi_{1} z^{d-1}+\ldots
$$

then

$$
P_{1}(v)=v+\frac{1}{d} \Phi_{1},
$$

which shows that

$$
\Phi^{(n)}(v)+\frac{1}{d} \Phi_{1}=P_{d^{n}}(v),
$$

in agreement with Theorem 11.

We now come to the family of orthogonal polynomials $P_{n}^{\infty}(v)$ with respect to the measure $\gamma^{\infty}(v)$, and we know already a subset of this family, namely those of degree $d^{n}$. In order to study the whole family, we analyse the properties of the coefficients of the three term recursion relation (II.15), and we have the following:

Theorem 15. The coefficient of the three term recursion relation (II.15) satisfied by the polynomials $P_{n}(v)$ orthogonal with respect to a measure $\gamma^{\infty}(v)$ invariant under a monic polynomial transformation $F$, fulfill the following system of equations:

$$
\left.\begin{array}{rl}
R_{n+1} & =R_{n d-d+2} R_{n d-d+3} \ldots R_{n d+1}, \\
\Delta(d n-d+1, d-1) & =\Delta(d n+1, d-1), \\
\Delta(d n, d)-\Delta(d n-d+1, d-2) R_{d n+1} & =F(v)-B_{n+1},
\end{array}\right\}
$$

where the polynomial $\Delta(p, q)$ of degree $q$ in $v$ is defined in Eq. (IV.14). These equations allow us to compute recursively the coefficients $R_{n}$ and $B_{n}$.

This theorem is a straightforward consequence of Theorems 4 and 6 , using (VI.4). In order to initialize the recursion relations (VII.15), we need those $R_{n}$ and 
$B_{n}$ which are contained in $\Delta(1, d-1)$, that is $B_{2}, B_{3} \ldots B_{d}$, and $R_{3}, R_{4} \ldots, R_{d}$. They are computed from the series expansion of $g(z)$. It is however useful to observe that these coefficients are also contained in the continued fraction expansion of $g^{\infty}(z)$ :

$$
\begin{aligned}
g^{\infty}(z)=\frac{1}{\left(1-B_{1} z\right)-\frac{R_{2} z^{2}}{\left(1-B_{2} z\right)-R_{3} z^{2}}} & \\
\cdots & -\frac{R_{p} z^{2}}{\left(1-B_{p} z\right)-R_{p+1} z^{2}}
\end{aligned}
$$

In fact the situation is simpler when $F \in S_{d}$, because in this case, the coefficients $B_{1}$, $B_{2} \ldots B_{d}, R_{2} \ldots R_{d}$ can be computed from the continued fraction expansion of

$$
R_{(1)}^{F}(z)=\frac{1}{d z} \frac{F^{\prime}(1 / z)}{F(1 / z},
$$

which is the $[d-1 / d]$ Padé approximant to $g^{\infty}(z)$. Of course in the continued fraction expansion of $R_{(1)}^{F}$, we must replace $R_{d n+1}$ by zero. More generally, the continued fraction expansion of:

$$
R_{(n)}^{F}(z)=\frac{1}{d^{n} z} \frac{F^{(n)^{\prime}}(1 / z)}{F^{(n)}(1 / z)}
$$

has the same coefficients as (VII.16) up to order $d^{n}$ which means that in $R_{(n)}^{F}, R_{d^{n}+1}$ is replaced by zero.

The continued fraction approach allows us to clarify the question we have left aside at the end of Sect. IV : our arguments are valid as long as no $R_{p}$ vanish. When some $R_{p}$ vanish, the system of orthogonal polynomials can either be complete, or some polynomials are missing. Some Padé approximants to $g^{\infty}(z)$ may also be missing. Here we know that the $\left[d^{n}-1 / d^{n}\right]$ Padé approximants always exist, and this is an indication that in the generic situation no $R_{p}$ will vanish. In fact one can solve the system (VII.15) formally in expressing the coefficients $R_{p}$ and $B_{p}$ as functions of the external parameters, namely the coefficients of the polynomial $F(v)$. Thus $R_{p}$ and $B_{p}$ turn out to be rational fractions in these coefficients. The set of orthogonal polynomials will become singular when the denominators of $R_{p}$ vanish. But as meromorphic functions of the coefficients of $F$, the system of equations always makes sense.

\section{An Illustrative Example: The Quadratic Case}

We consider the polynomial transformation:

$$
F(z)=z^{2}+\lambda, \quad d=2 .
$$

The function $g^{\infty}(z)$ is the solution of

$$
g^{\infty}(z)=\frac{1}{1+\lambda z^{2}} g^{\infty}\left(\frac{z^{2}}{1+\lambda z^{2}}\right),
$$


to which corresponds the measure

which satisfies

$$
\gamma^{\infty}(v)=\frac{1}{v} g^{\infty}\left(\frac{1}{v}\right)
$$

$$
\gamma^{\infty}(v)=v \gamma^{\infty}\left(v^{2}+\lambda\right)
$$

Between the orthogonal polynomials $P_{n}(v)$ with respect to $\gamma^{\infty}(v)$ and the iterated mappings $F^{(n)}(v)$, we have the relation

$$
P_{2^{n}}(v)=F^{(n)}(v)
$$

The polynomials $P_{n}$ satisfy

$$
P_{n}\left(v^{2}+\lambda\right)=P_{2 n}(v)
$$

Using Theorem 15, we get

$$
\left.\begin{array}{rl}
R_{n+1} & =R_{2 n} R_{2 n+1}, \\
R_{2 n+1}+R_{2 n+2} & =-\lambda, \\
B_{n} & =0 .
\end{array}\right\}
$$

The Eqs. (VIII.7) permit the recursive computation of the $R_{p}$ starting from

$$
R_{2}=-\lambda, \quad R_{3}=1
$$

The preceding results are equivalent to those already obtained in [16] and [17] from the polynomial map $(v-q)^{2}$, with the slight modification that the present $R_{n}$ is equal to $R_{n-1}$ in the above references. We have used in the present paper notations following Szegö's classical prescriptions [19], for the recursion relation

$$
P_{n}=\left(v-B_{n}\right) P_{n-1}-R_{n} P_{n-2} .
$$

Since all $B_{n}$ vanish, the $P_{n}$ are alternatively odd or even according as $n$ is odd or even. The case of $\lambda$ real and less than -2 was treated in detail in [17]. (Here what we call $\lambda$ corresponds to $-q$ in that reference.) In this case, the Julia set is a real Cantor set made of the accumulation points of the sequences

$$
|\lambda| \pm \sqrt{|\lambda| \pm \sqrt{|\lambda|+\ldots+\sqrt{|\lambda|}}}
$$

and the values of $R_{n}(\lambda)$ have the following properties established in [17]:

Theorem 16. For $\lambda$ real $<-2$, we have

$$
\begin{aligned}
\text { i) } & 0<R_{2 n+1}<R_{n+1} \text { and } 0<R_{2 n+1} \leqq 1 \text {, } \\
\text { ii) } & \lim _{k \rightarrow \infty} R_{\left(p 2^{k}+1\right)}=0, \\
\text { iii) } & \lim _{k \rightarrow \infty} R_{\left(p 2^{k}+s\right)}=R_{s} .
\end{aligned}
$$

These results allow us to determine the set of values $C_{\lambda}$ taken by the $R_{n}(\lambda)$ for varying $n$. Each point of $C_{\lambda}$ is an accumulation point, and $C_{\lambda}$ is made of disjoint pieces. 
We also mention

Theorem 17. The mapping $v^{2}+\lambda$ has a superstable cycle of order $k$ when $\lambda$ is solution of the polynomial equation

$$
\prod_{h=1}^{2(k-1)} R_{2 h}(\hat{\lambda})=0 .
$$

The proof starts from an argument due to Myrberg [11]; the mapping has a superstable cycle of order $k$ if $\lambda$ is such that

which reads

$$
F^{(k)}(v=0)=0,
$$

$$
P_{2^{k}}(0)=0
$$

However we see from (VIII.9) that

$$
P_{k}(0)=-R_{k} P_{k-2}(0)
$$

Therefore we get

$$
P_{2^{k}}(0)=R_{2} R_{4} \ldots R_{\left(2^{k}-2\right)} R_{2^{k}} .
$$

This proves the theorem. Notice that a priori $R_{p}$ is a rational fraction in $\lambda$, but cancellations occur and (VIII.18) shows that (VIII.14) is actually a polynomial equation in $\lambda$. We see that in the quadratic case, superstable cycles are associated with singular orthogonal polynomial sets, having some vanishing $R_{n}$ coefficients. We do not know how to generalize Theorem 17 to higher degree polynomial mappings.

\section{Conclusion}

Our Theorem 13 and 14 establish a rather unexpected connection between polynomial mappings and orthogonal polynomials on a complex contour encircling the Julia set. We think that one of the most promising consequences of this connection is the three term recursion relation associated with the orthogonal polynomials

$$
P_{n}(v)=\left(v-B_{n}\right) P_{n-1}(v)-R_{n} P_{n-2}(v) .
$$

Now we interpret this recursion relation in $n$ for fixed $v$. This is a linear relation, permitting the computation of $P_{n}(v)$ from lower order polynomials. The question is: what is the asymptotic behaviour of this recursion relation when $n$ tends to infinity? If we knew the answer to this question, we would immediately obtain the behaviour of the iterated mapping, by now considering $n$ in (IX.1) as being equal to $2^{P}$. The difficult problem, of course, is the knowledge of the dependence on $n$ of the $B_{n}$ and $R_{n}$. This is the reason for the importance of Theorem 15 which allows us to compute recursively these coefficients, through a product of linear and homographic transformations. We understand (IX.1) in some sense as a discrete analogous version of the classical inverse scattering method for solving nonlinear partial differential equations [22]. We are looking forward to studying in detail the behaviour of the coefficients $R_{n}$ and $B_{n}$ which play in some sense the role of a potential in the usual second order differential equation. The connection with the 
one dimensional Schrödinger equation has recently been made in reference [23] for the quadratic case, producing an interesting, almost periodic Schrödinger operator with singular continuous spectrum and chaotic extended states. Note also that the Jacobi matrix $H$, defined as in (V.35) for the set of orthogonal polynomials assocated with $\gamma^{\infty}$, satisfies an algebraic equation similar to (V.39)

$$
D F(H)=H D \text {. }
$$

As noticed in [23], this equation may lead to a promising interpretation in the framework of renormalization group theory.

After our work was completed, we received a paper [24] devoted to invariant measures on Julia sets. Although some of the results presented there are parallel to ours, we noticed the following differences: they consider orthogonal polynomials with respect to a hermitian scalar product, which does not allow contour deformations in the complex plane. Here we consider general polynomial transformations on measures and not only their fixed points. Of course, the results of both approaches coincide when the Julia set is real.

\section{Appendix}

Proof of Theorem 3. We rewrite Eq. (IV.9) for $k=n d+1, n d+2, \ldots, n d+d$, in matrix form (for $d \geqq 3$ ):

where

$$
\sum_{l=1}^{d-1}[M(d n+1, d-1)]_{k l} V_{l}^{(n)}(v)=W_{k}^{(n)}(v),
$$

But (A.1) can be inverted, and we get

$$
V_{l}^{(n)}(v)=\sum_{m}[M(d n+1, d-1)]_{l m}^{-1} W_{m}^{(n)}(v)
$$

which allows us to express $P_{d n+l}$ in terms of $P_{d n}$ and $P_{d n+d}$ exclusively, due to the particular form $W_{k}^{(n)}(v)$. We are especially interested in computing $P_{d n+1}$ and $P_{d n+(d-1)}$. However, expressing the inverse matrix elements in terms of minor determinants gives

$$
\begin{aligned}
{[M(d n+1, d-1)]_{1,1}^{-1} } & =\frac{\Delta(d n+2, d-2)}{\Delta(d n+1, d-1)}, \\
{[M(d n+1, d-1)]_{1,(-1)}^{-1} } & =\frac{1}{\Delta(d n+1, d-1)}, \\
{[M(d n+1, d-1)]_{(d-1), 1}^{-1} } & =\frac{R_{d n+3} R_{d n+4} \ldots R_{d n+d}}{\Delta(d n+1, d-1)}, \\
{[M(d n+1, d-1)]_{(d-1),(d-1)}^{-1} } & =\frac{\Delta(d n+1, d-2)}{\Delta(d n+1 . d-1)} .
\end{aligned}
$$


from which we get

$$
\begin{aligned}
P_{d n+1}(v) & =\frac{\Delta(d n+2, d-2)}{\Delta(d n+1, d-1)} R_{d n+2} P_{d n}(v)+\frac{1}{\Delta(d n+1, d-1)} P_{d n+d}(v), \\
P_{d n+d-1}(v) & =\frac{R_{d n+2} R_{d n+3} \ldots R_{d n+d}}{\Delta(d n+1, d-1)} P_{d n}(v)+\frac{\Delta(d n+1, d-2)}{\Delta(d n+1, d-1)} P_{d n+d}(v) .
\end{aligned}
$$

Now using (II.15) and (IV.10), we have

$$
P_{d n+1}(v)=A_{d n+1} P_{d n}(v)-R_{d n+1} P_{d n-1}(v) .
$$

We use (A.11) to express $P_{d n+1}$, and (A.12) to express $P_{d n-1}$, after changing $n$ into $n-1$. Thus we get from (A.13)

$$
\begin{aligned}
& \Delta(d n-d+1, d-1) P_{d n+d}(v)=\left\{\Delta ( d n - d + 1 , d - 1 ) \left(\Delta(d n+1, d-1) A_{d n+1}\right.\right. \\
& \left.\quad-\Delta(d n+2, d-2) R_{d n+2}\right) \\
& \left.\quad-\Delta(d n-d+1, d-2) \Delta(d n+1, d-1) R_{d n+1}\right\} P_{d n}(v) \\
& \quad-R_{d n-d+2} R_{d n-d+3} \ldots R_{d n+1} \Delta(d n+1, d-1) P_{d n-d}(v) .
\end{aligned}
$$

Now using (IV.21) we get (IV.17) and (IV.20) which proves Theorem 3.

Proof of Theorem 6. Given all $B_{n}$ and $R_{n} \neq 0$, as well as the polynomial $F(v)$, we shall show that a recursive procedure permits the computation of all $B_{n}^{F}$ and $R_{n}^{F}$ starting from those for which $n \leqq d$. Suppose that we have been able to compute $B_{m}^{F}$ and $R_{m}^{F}$ up to order $m=n d$ included. Then the polynomial $\Delta(n d-d+1, d-1)$ is built only with known quantities. From (IV.29) we get $R_{n d+1}^{F}$, and from (IV.28) and (IV.30) we see that $\Delta^{F}(d n+1, d-1)$ and $\Delta^{F}(d n, d)$ become known polynomials in $v$ : more precisely, we have to solve the following equations:

$$
\left\{\begin{aligned}
\Delta^{F}(d n+1, d-1) & =\Delta^{F}(d(n-1)+1, d-1) \\
\Delta^{F}(d n, d) & =\Delta^{F}(d n-d+1, d-2) R_{d n+1}^{F}-F(v)+B_{n+1} .
\end{aligned}\right.
$$

The unknown $B_{d n+1}^{F}, B_{d n+2}^{F}, \ldots, B_{d n+d}^{F}, R_{d n+2}^{F}, R_{d n+3}^{F}, \ldots, R_{d n+d}^{F}$ are only present in the left hand sides. The right hand sides of the preceding equations (A.15) and (A.16), are made with known quantities. Since $\Delta(p, q)$ is a monic polynomial of degree $q$, there are in fact $(d-1)$ constraints in (A.15) and $d$ constraints in (A.16) so, in principle we can solve for the $(2 d-1)$ unknowns. However we can multiply (A.15) by $-A_{d n+1}^{F}$ and then add the result with (A.16) and we get

$$
\begin{aligned}
& \Delta^{F}(d n, d)-A_{d n+1}^{F} \Delta^{F}(d n+1, d-1) \\
& \quad=\Delta^{F}(d n-d+1, d-2) R_{d n+1}^{F}-F(v)+B_{n+1}-A_{d n+1}^{F} \Delta^{F}(d(n-1)+1, d-1) .
\end{aligned}
$$

From (IV.21) the left hand side of (A.17) is equal to $-\Delta^{F}(d n+2, d-2) R_{d n+2}^{F}$, a polynomial of degree $(d-2)$. Then the terms of degree $d$ and $d-1$ have to cancel in (A.17). The terms of degree $d$ cancel automatically because $\Delta^{F}, F$, and $A_{d n+1}^{F}$ are all monic polynomials. The term of degree $d-1$ allows us to compute $B_{d n+1}^{F}$ in terms of the previously computed quantities. Therefore we also express as a function of 
the previously known quantities the combination

$$
\begin{aligned}
-\Delta(d n+2, d-2) R_{d n+2}^{F}= & \Delta^{F}(d n-d+1, d-2) R_{d n+1}^{F}-F(v)+B_{n+1} \\
& -\left(v-B_{d n+1}^{F}\right) \Delta^{F}(d n-d+1, d-1) .
\end{aligned}
$$

The term of degree $(d-2)$ of the preceding equation allows us to solve for $R_{d n+2}^{F}$. Simultaneously we express the polynomial $\Delta^{F}(d n+2, d-2)$ in terms of known quantities provided $R_{d n+2}^{F}$ does not vanish. The situation is now as follows. We have expressed in terms of known quantities $\Delta^{F}(d n+2, d-2)$ and $\Delta^{F}(d n+1, d-1)$ which are expressions containing now $(2 d-3)$ unknows, namely: $B_{d n+2}^{F}$, $B_{d n+3}^{F}, \ldots, B_{d n+d}^{F}, R_{d n+3}^{F}, R_{d n+4}^{F} \ldots R_{d n+d}^{F}$. We have for these equations a system of the following from:

$$
\left\{\begin{array}{l}
\Delta^{F}(d n+2, d-2)=\text { known }, \\
\Delta^{F}(d n+1, d-1)=\text { known } .
\end{array}\right.
$$

Therefore we are back to the same system of equations as (A.15) and (A.16) with the following modification: the indices of $\Delta^{F}$ in the left hand side have been increased by one for the first and decreased by one for the second, the number of unknowns has been decreased by two. Clearly the procedure applied to the systems (A.15) and (A.16) can be repeated to the systems (A.19) and (A.20), which will solve for $B_{d n+2}^{F}, R_{d n+3}^{F}$, and get $\Delta^{F}(d n+3, d-3)$ and $\Delta^{F}(d n+2, d-2)$, decreasing once more the number of unknowns by two. Clearly the procedure can be repeated until we get $B_{d n+d}^{F}$ from $\Delta^{F}(d n+d-1,1)$. The procedure will break down if any $R_{m}^{F}$ vanishes: we have thus completed the proof of Theorem 6.

Acknowledgements. We want to thank Drs. M. F. Barnsley, B. Duplantier, J. S. Geronimo, and A. Pandey for fruitful discussions. Our work owes very much to M. L. Mehta with whom the quadratic case has already been studied. We have also benefited from the experience of B. Derrida on the subject, and we thank him especially for his contribution which allowed us to write Eq. (VI.I) and Theorem 6. The operator version (V.39) and (IX.2) has been obtained in collaboration with J. Bellissard.

\section{References}

1. Ruelle, D., Takens, F.: On the nature of turbulence. Commun. Math. Phys. 20, 167-192 (1971)

2. Eckmann, J.P.: Roads to turbulence in dissipative dynamical systems. Rev. Mod. Phys. 53, 653-654 (1981)

3. Collet, P., Eckmann, J.P. : Iterated maps on the interval as dynamical systems. Progress in physics, Vol. 1, Boston: Birkhäuser 1980

4. May, R.M. : Simple mathematical models with very complicated dynamics. Nature 261, 459-467 (1976)

5. Feigenbaum, M.: Quantitative universality for a class of nonlinear transformations. J. Stat. Phys. 19, 25-52 (1978) and: The universal metric properties of nonlinear transformations. J. Stat. Phys. 21, 669-706 (1979)

6. See for instance: Libchaber, A., Maurer, J.: Une expérience de Rayleigh-Bénard de géométrie réduite; multiplication, accrochage, et démultiplication de fréquences. J. Phys. (Paris) Coll. C3, 51-56 (1980)

7. Derrida, B., Gervois, A., Pomeau, Y.: Universal properties of bifurcations of endomorphisms. J. Phys. A: Math. Gen 12, 269-296 (1979)

8. Julia, G. : Mémoire sur l'itération des fonctions rationnelles. J. Math., Ser. 7 (Paris) 4, 47-245 (1918)

9. Fatou, P.: Sur les équations fonctionelles. Bull. Soc. Math. France 47, 161-271 (1919); 48, 33-94 (1920) ; 48, 208-314 (1920) 
10. Brolin, H.: Invariants sets under iteration of rational functions. Ark. Mat. 6, 103-144 (1965)

11. Myrberg, P.J.: Sur l'iteration des polynômes réels quadratiques. J. Math. Pures Appl. 41, 339-351 (1962)

12. Douady, A., Hubbard, J.H.: Iteration des polynômes quadratiques complexes. C.R. Acad. Sci. (Paris) 294, Série I, 123-126 (1982)

13. Ruelle, D.: Application conservant une mesure absolument continue par rapport à $d x$ sur $[0,1]$. Commun. Math. Phys. 55, 47-51 (1977)

14. Barnsley, M.F., Bessis, D., Moussa, P.: The diophantine moment problem and the analytic structure in the activity of the ferromagnetic Ising model. J. Math. Phys. 20, 535-546 (1979)

15. Moussa, P.: Problème diophantien des moments et modèle d'Ising. Accepted for publication in Annales de l'Institut Henri Poincaré

16. Bessis, D., Mehta, M.L., Moussa, P.: Polynômes orthogonaux sur des ensembles de Cantor et iterations des transformations quadratiques. C.R. Acad. Sci. (Paris) 293, Série I, 705-708 (1981)

17. Bessis, D., Mehta, M.L., Moussa, P.: Orthogonal polynomials on a family of Cantor sets and the problem of iterations of quadratic mappings. Lett. Math. Phys. 6, 123-140 (1982)

18. Barnsley, M.F., Geronimo, J.S., Harrington, A.N.: On the invariant sets of a family of quadratic maps. Commun. Math. Phys. 88, 479-501 (1983)

19. Szegö, G.: Orthogonal polynomials. American Mathematical Society Colloquium Publication 23, 1939

20. Baker, G.A. Jr.: The essential of Padé approximants. New York: Academic Press 1978

21. Titchmarsh, E.C.: The theory of functions, p. 168. Oxford: Oxford University Press 1932

22. See for instance: Solitons, Bullough, R.K., Caudrey, P.J. (eds.): Topics in current physics, Vol. 17. Berlin, Heidelberg, New York: Springer 1980

23. Bellissard, J., Bessis, D., Moussa, P.: Chaotic states of almost periodic Schrödinger operators. Phys. Rev. Lett. 49, 701-704 (1982)

24. Barnsley, M.F., Geronimo, J.S., Harrington, A.N.: Orthogonal polynomials associated with invariant measures on Julia sets. Bull. Am. Math. Soc. 7, 381-384 (1982)

Communicated by O.E. Lanford

Received March 22, 1982; in revised form November 3, 1982 
